\title{
Energy consumption analysis of modulation schemes in IEEE 802.15.6-based wireless body area networks
}

\author{
K. S. Deepak ${ }^{1 *}$ and A. V. Babu ${ }^{1,2}$
}

\begin{abstract}
Selection of the most energy-efficient modulation scheme is vital for wireless body area networks (WBANs) for improving the network lifetime. In this paper, we investigate the performance of various modulation schemes specified by IEEE 802.15.6 WBAN standard, in terms of energy consumed for successfully transferring one packet from the sensor node to the hub. Assuming a contention-free MAC protocol, we present an analytical model for computing the energy consumption for a given modulation scheme both for uncoded transmission as well as transmission using Bose-Chaudhuri-Hocquengham (BCH) codes; the coding scheme specified by IEEE 802.15.6 for WBAN. The following communication scenarios specific to a WBAN are considered: (i) in-body communication between invasive devices and hub and (ii) on-body communication among non-invasive devices and hub with line-of-sight (LOS) and non-LOS (NLOS) channels. The results show that the use of higher order differential phase shift keying (D8PSK)-based modulation schemes results in significant reduction of total energy consumption. When $\mathrm{BCH}$ codes with various code rate specifications are used, total energy consumption remains lower for the higher order modulation schemes. The energy consumption is further reduced when non-coherent frequency shift keying (NC-MFSK) is employed as an alternate modulation scheme instead of differential PSK schemes.
\end{abstract}

Keywords: Energy consumption, IEEE 802.15.6, Wireless body area networks, Modulation schemes

\section{Introduction}

Wireless body area networks (WBANs) consist of a number of low-power sensor nodes that are attached to the body surface, implanted inside or around the body, that monitor vital physiological parameters and transmit the data to a central device known as hub. The hub in turn transfers the gathered data to a centralized repository through suitable access networks [1]. Broadly, WBANs can be classified as in-body (implantable) and on-body (wearable) systems. While the former allows RF communication between invasive devices and hub, the latter supports RF communication among non-invasive devices and hub.

One of the key challenges in WBAN research is the design of energy-efficient communication protocols for

*Correspondence: p110043ec@nitc.ac.in

${ }^{1}$ Research scholar, Department of Electronics and Communication Engineering, National Institute of Technology, Calicut, Kerala 673601, India Full list of author information is available at the end of the article improving the network lifetime. Another critical aspect of WBANs is the reliability, which is highly application dependent [2]. For example, medical applications that involve real-time collection of patient's vital physiological information require almost $100 \%$ reliability [3]. It has been reported that the wireless link between the sensor and the hub experiences severe attenuation of the order of 50 to $100 \mathrm{~dB}$. Further, WBANs experience deep fading effects that can last much longer (10-300 ms) than that experienced in cellular networks and severe shadowing effects that can result in very high outage probability [4]. At the same time, to reduce the heating of human body tissues and interference to other biosensor devices operating in the same frequency band, there is a limit on maximum transmit power that can be used in such devices [3]. For instance, in the case of in-body communication that occurs in the medical implantable communication service (MICS) band of 402-405 MHz, the effective isotropic radiated power (EIRP) is specified to be 25 microW $(-16 \mathrm{dBm})$. 
The IEEE 802.15 Task Group 6 has recently approved the PHY and MAC specifications for WBANs [5]. It defines a MAC layer in support of three PHY layers: narrow band (NB), ultrawide band (UWB), and human body communications (HBC). The selection of each PHY depends on the application requirements. The standard specifies the use of Bose-Chaudhuri-Hocquengham $(\mathrm{BCH})$ code $(n=63, k=51, t=2)$ for data transmission in the NB PHY. The physical layer protocol data unit (PPDU) of NB PHY contains physical layer convergence protocol (PLCP) preamble, PLCP header, and a PHY service data unit (PSDU) as shown in Fig. 1. The PSDU consists of MAC header, MAC frame body, and frame check sequence (FCS). The PSDU is encoded using binary $\mathrm{BCH}$ code and spread/interleaved before transmission. Table 1 shows the details of the frequency bands specified by IEEE 802.15 .6 and the modulation schemes applicable for these seven frequency bands [5]. In this paper, we consider in-body communication in the frequency band $402-405 \mathrm{MHz}$ and on-body communication that takes place in the $2.360-2.400-\mathrm{GHz}$ and $2.400-2.483-\mathrm{GHz}$ bands. Table 2 shows the data rate-dependent modulation and channel coding parameters for PLCP header and PSDU in these frequency bands [5].

The focus of this paper is on the energy consumption analysis of various modulation schemes corresponding to the NB PHY as specified in the IEEE 802.15.6 standard. The motivation behind $M$-ary PSK is to increase the bandwidth efficiency by a factor $\log _{2} M$, where $M$ is the constellation size. Further, the use of differential modulation schemes avoids the need for coherent carrier reference at the receiver. This results in simpler systems suitable for WBANs. The IEEE 802.15.6 standard specifies the following digital modulation schemes for the NB PHY: differential binary phase shift keying (DBPSK), differential quadrature phase shift keying (DQPSK), differential 8 phase-shift keying (D8PSK), and Gaussian minimum shift keying (GMSK). From Table 1, it is clear that, out of the seven frequency bands allowed for WBAN services, GMSK modulation is specified in only one frquency band, i.e., $420-450-\mathrm{MHz}$ band. The $420-450-\mathrm{MHz}$ band is applicable for WBAN NB PHY layer services in Japan, whereas the two frequency bands that we consider in this paper are applicable for WBAN services worldwide. Further, even though GMSK improves the spectral efficiency, it requires higher power level than QPSK in order to transmit reliably the same amount of data [6]. Accordingly, in this paper, we focus on energy consumption analysis of the differential PSK modulation schemes and do not focus on GMSK. At the transmitter, differential encoding of the input binary bits is followed by phase shift keying. The differential encoded information is carried in the phase transitions between symbols. The constellation mapping details for the various differential modulation schemes are given in Tables 3, 4, and 5 [5].

Selection of the most energy efficient modulation scheme is important in the design of energy constrained WBAN to improve the lifetime of nodes. We also investigate the energy consumption performance of noncoherent $M$-ary frequency shift keying (NC-MFSK) in WBAN. We consider NC-MFSK, as an alternate modulation scheme due to the following advantages it offers [7]: (i) it is power efficient which results in lower transmission energy requirement for a given BER performance, (ii) it is non-coherent which avoids the need for carrier synchronization, (iii) it reduces the start-up time using direct modulation approach which avoids the need for mixer and digital to analog converter. The above advantages are achieved at the expense of increased bandwidth requirement. However, for low data rate applications and when energy constraint is more important than bandwidth

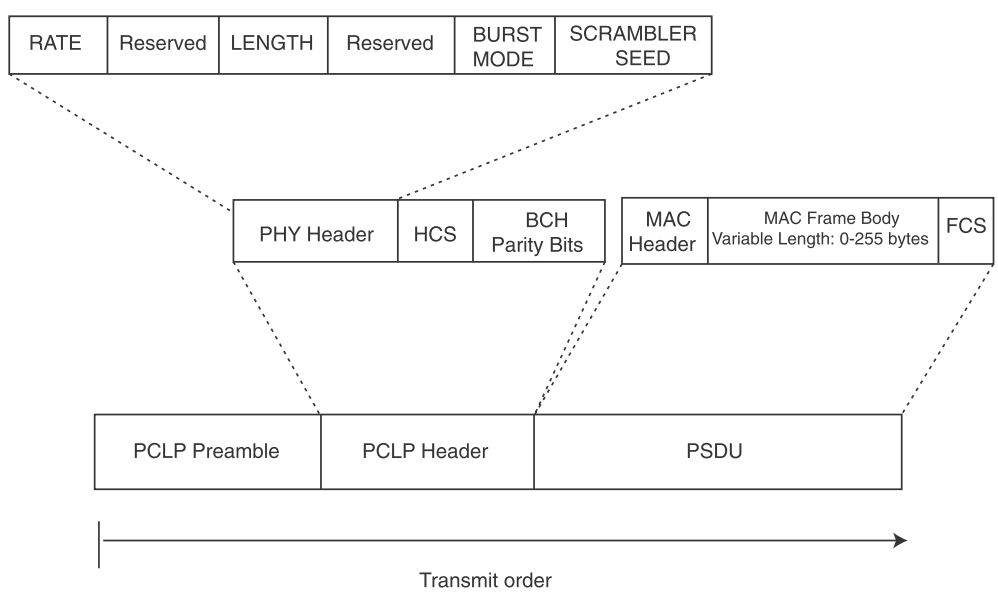

Fig. 1 The PPDU structure of narrow band PHY of IEEE 802.15.6 standard 
Table 1 Frequency bands for IEEE802.15.6 WBAN [5]

\begin{tabular}{lllll}
\hline $\begin{array}{l}\text { Band } \\
(\mathrm{MHz})\end{array}$ & Modulation & $\begin{array}{l}\text { Symbol rate } \\
(\mathrm{ksps})\end{array}$ & $\begin{array}{l}\text { Code rate } \\
\text { (of data) }\end{array}$ & $\begin{array}{l}\text { Maximum data rate } \\
\text { (kbps) }\end{array}$ \\
\hline $402-405$ & DBPSK, DQPSK, D8PSK & 187.5 & $51 / 63$ & 455.4 \\
$420-450$ & GMSK & 187.5 & $51 / 63,1 / 1$ & 187.5 \\
$863-870$ & DBPSK, DQPSK, D8PSK & 250 & $51 / 63$ & 607.1 \\
$902-928$ & DBPSK, DQPSK, D8PSK & 250 & $51 / 63$ & 607.1 \\
$950-958$ & DBPSK, DQPSK, D8PSK & 250 & $51 / 63$ & 607.1 \\
$2360-2400$ & DBPSK, DQPSK & 600 & $51 / 63$ & 971.4 \\
$2400-2483.5$ & DBPSK, DQPSK & 600 & $51 / 63$ & 971.4 \\
\hline
\end{tabular}

constraint, NC-MFSK modulation scheme with lower order constellation (lower $M$ ) is an attractive option.

Several papers have appeared in the literature that deal with energy efficiency and reliability issues in WBANs [8-15]. In [8], the author considers optimal power allocation to improve the energy efficiency of WBANs with constraints on outage probability. In [9], outage performance and energy efficiency of direct transmission and single and multi-relay cooperation schemes are analysed in the context of WBANs. Energy efficiency as well as optimal packet size are analysed in [10] for cooperative WBANs based on a realistic channel model. The energy efficiency of IEEE 802.15.6-compliant WBAN has been analysed in [11] for the scheduled access mode. In [12], Arrobo and Gitlin propose cooperative network coding to improve reliability of WBANs. Oh et al. [13] propose a new type of phase shift keying modulation scheme, namely phase silence shift keying (PSSK), for high data rate implantable medical devices and show that the bit error rate (BER) of an 8-PSSK is lower than that of various sinusoidal carrierbased modulation schemes. In [14], Elias investigates the optimal design of wireless body area networks by studying the joint data routing and relay positioning problem, in order to increase the network lifetime. In [15], Zhou et al. address energy-efficient resource allocation in WBANs.
Different from all the above, the focus of the present paper is on the evaluation of energy consumption corresponding to various modulation schemes specified by IEEE 802.15.6 for the NB PHY layer operation, taking into account both in-body and on-body channel models.

The major contributions of our paper are as follows: We investigate the performance of different modulation schemes for IEEE 802.15.6-complaint WBAN. We evaluate the performance of the modulation schemes in terms of total energy consumed per sensor node for successfully delivering a packet to the hub. We also evaluate the impact of code rate on the total energy consumption. We present analytical models to quantify the energy consumed per successful packet transmission. The rest of the paper is organized as follows. Section 2 describes the energy consumption analysis. Section 3 describes the results and discussions. Finally, Section 4 concludes the paper.

\section{Energy consumption analysis}

In this section, we present the energy consumption analysis of various modulation schemes in WBAN. We assume a WBAN with star topology in which data packets are transferred directly between the sensor node and the hub. At the MAC layer, we assume a contention-free MAC scheme such as scheduled up link/down link access

Table 2 Modulation parameters for PLCP header and PSDU [5]

\begin{tabular}{|c|c|c|c|c|c|c|}
\hline $\begin{array}{l}\text { Frequency } \\
\text { bands }\end{array}$ & $\begin{array}{l}\text { Packet } \\
\text { component }\end{array}$ & Modulation (M) & $\begin{array}{l}\text { Symbol rate }=1 / \pi \mathrm{s} \\
\text { (ksps) }\end{array}$ & Code rate $(\mathrm{k} / \mathrm{n})$ & $\begin{array}{l}\text { Spreading factor } \\
(S)\end{array}$ & $\begin{array}{l}\text { Information data rate } \\
\text { (kbps) }\end{array}$ \\
\hline $2360 \mathrm{MHz}$ & PLCP header & $\pi / 2$-DBPSK $(M=2)$ & 600 & $19 / 31$ & 4 & 91.9 \\
\hline$-2400 \mathrm{MHz}$ & PSDU & $\pi / 2-\operatorname{DBPSK}(M=2)$ & 600 & $51 / 63$ & 4 & 121.4 \\
\hline and $2400 \mathrm{MHz}$ & PSDU & $\pi / 2-\mathrm{DBPSK}(M=2)$ & 600 & $51 / 63$ & 2 & 242.9 \\
\hline - $2483.5 \mathrm{MHz}$ & PSDU & $\pi / 2-\mathrm{DBPSK}(M=2)$ & 600 & $51 / 63$ & 1 & 485.7 \\
\hline \multirow[t]{2}{*}{ (on-body) } & PSDU & $\pi / 4-\mathrm{DQPSK}(M=4)$ & 600 & $51 / 63$ & 1 & 971.4 \\
\hline & PLCP header & $\pi / 2$-DBPSK $(M=2)$ & 187.5 & $19 / 31$ & 2 & 57.5 \\
\hline $402 \mathrm{MHz}$ & PSDU & $\pi / 2-\mathrm{DBPSK}(M=2)$ & 187.5 & $51 / 63$ & 2 & 75.9 \\
\hline$-405 \mathrm{MHz}$ & PSDU & $\pi / 2$-DBPSK $(M=2)$ & 187.5 & $51 / 63$ & 1 & 151.8 \\
\hline \multirow[t]{2}{*}{ (in-body) } & PSDU & $\pi / 4-D Q P S K ~(M=4)$ & 187.5 & $51 / 63$ & 1 & 303.6 \\
\hline & PSDU & $\pi / 8-\mathrm{D} 8 \mathrm{PSK}(M=8)$ & 187.5 & $51 / 63$ & 1 & 455.4 \\
\hline
\end{tabular}


Table 3 Constellation mapping for DBPSK [5]

\begin{tabular}{ll}
\hline$b(n)$ & $\phi$ \\
\hline 0 & 0 \\
1 & $\pi$ \\
\hline
\end{tabular}

mechanism [5]. For the energy consumption analysis, we consider the energy consumption model that takes into account the transmit energy, the start-up energy, and the receive energy [16]. In order to minimize the energy consumption, the sensor node in WBAN normally stays in the sleep state by turning off sub-systems like crystal oscillator when not required and wakes up for transmitting the data. The node consumes transient energy to wake up from sleep state to reach the active state. The total energy consumption consists of following components: (i) energy required for the sensor node to wake up from the sleep state; (ii) energy required to transmit the data; (iii) energy consumed during the data reception; and (iv) energy cost for decoding the data. Accordingly, one of the most important goals in the design of WBANs is to achieve minimum energy communication. The development of a physical layer-specific energy consumption model would be a crucial step towards the design of energy-efficient physical layer for WBANs. We assume that a data packet of length $\mathrm{L}$ bits is to be transmitted to the hub. The data packet is divided into $\left\lceil\frac{L}{k}\right\rceil$ blocks each of length $k$ bits.

The battery is currently the largest component of the implantable medical device. The limited capacity of the batteries used by the sensor nodes restricts the lifetime of the node. At the same time, battery replacement is not always feasible since it may jeopardize the patient's health. In the case of sensor nodes implanted inside the human body, replacement of battery would require surgical procedures. Recently, significance advancements have been made in the areas of wireless charging and energy harvesting to power implanted medical devices. Energy harvesting has proven to be an effective alternative to pure battery power in medical implant applications given the ability to produce power from nano-watts to several milli-watts, which is in the power consumption range of the most common implantable medical devices today. Different types of energy can be harvested from a variety of sources. For example, solar energy can be collected from the environment. Kinetic energy can be derived from the motion

Table 4 Constellation mapping for DQPSK [5]

\begin{tabular}{lll}
\hline$b(2 n)$ & $b(2 n+1)$ & $\phi$ \\
\hline 0 & 0 & $\pi / 4$ \\
0 & 1 & $3 \pi / 4$ \\
1 & 0 & $7 \pi / 4$ \\
1 & 1 & $5 \pi / 4$ \\
\hline
\end{tabular}

Table 5 Constellation mapping for D8PSK [5]

\begin{tabular}{llll}
\hline$b(3 n)$ & $b(3 n+1)$ & $b(3 n+2)$ & $\phi$ \\
\hline 0 & 0 & 0 & $\pi / 8$ \\
0 & 0 & 1 & $3 \pi / 8$ \\
0 & 1 & 0 & $7 \pi / 8$ \\
0 & 1 & 1 & $5 \pi / 8$ \\
1 & 0 & 0 & $15 \pi / 8$ \\
1 & 0 & 1 & $13 \pi / 8$ \\
1 & 1 & 0 & $9 \pi / 8$ \\
1 & 1 & 1 & $11 \pi / 8$ \\
\hline
\end{tabular}

of human activities, such as running or walking. The body can provide thermal energy. Several experimental demonstrations have been reported on energy harvesting strategies specific to human body based on thermoelectric and piezoelectric methods. However, these methods are anatomically specific, since energy harvesting works only in very specific parts of the body. In wireless charging, an electromagnetic field is created between two copper coils that are tuned to resonate at the same frequency, which then allows electricity to be transferred; these waves are in the near-field frequency spectrum. Near-field waves decay quickly and cannot penetrate deep into the body while far-field waves are not suitable for biological tissue applications as they either reflect off the body or are absorbed as heat. Recently, researchers at Stanford University have discovered an "in-between" technology that they call midfield wireless transfer, which can safely penetrate the body without interacting with biological tissue. The mid-field charging technology still needs to be tested in humans and receive safety and efficacy approval, so it could be several years before its adoption in commercial medical devices [17].

\subsection{Channel model}

In this section, we describe the channel model employed for our analysis. We consider two distinct communication scenarios: (i) in-body communication between implant sensor nodes (sensor nodes could be placed inside the human body immediately below the skin or deeper inside the body) and the hub; (ii) on-body communication between non-invasive sensor nodes and the hub. The non-invasive sensor node can have either LOS or NLOS communication with the hub. The human body has a complex structure which consists of tissues, muscles, and bones having different dielectric constants, thickness, and characteristic impedance. Since physical measurements and experimental studies inside the human body are extremely difficult, comprehensive characterization of in-body communication channel is tedious as compared to the on-body channel. In [18-20], authors develop a 
path loss model for in-body communication involving four near surface and two deep tissue implant application in male human body in the MICS frequency band. They report that traditional distance-dependent path loss model is applicable with deviation in $\mathrm{dB}$ caused by different materials such as the bone, muscle in the propagation path. The path loss models obtained in these studies have been adopted by the IEEE802.15 task group TG6 on body area networks in [21]. We have followed the same model for evaluating the performance of in-body communication in our paper. In [22], measurement results have been reported for the body surface to body surface CM3 (scenarios S4 and S5) channel model in the 2.4-GHz ISM band. The measurements have been done in a hospital room environment and an anechoic chamber. The following locations on the human body were considered (A - left wrist, B - left upper arm, C - left ear, D - head, E - shoulder, F - chest, G - right rib, H - left waist, I - thigh, $J$ - ankle). Through extensive measurements, the authors have established that the propagation model obeys the traditional distance-dependent model with appropriate parameters and lognormal shadow fading to represent the deviation. The path loss models obtained in this study have been adopted by the IEEE802.15 task group TG6 on body area networks in [21]. We have followed the same model for evaluating the performance of on-body communication (both LOS as well as NLOS) in our paper. The fading observed in on-body communication channels is typically the result of body shadowing and small-scale fading generated by the human body. Most of the empirical studies have reported that, since there are only a small number of multi-path components from the diffraction around the body, the small-scale fading effect is less severe with insignificant intersymbol interference from multipath [2]. Accordingly, we do not consider the fast fading component for our analysis and rely on a combined path loss and lognormal fading model. The path loss models for in-body communication at $402-405 \mathrm{MHz}$ and on-body LOS and NLOS communication at $2.4 \mathrm{GHz}$ are given by $[21,23,24]$ as

$$
L_{d}(\mathrm{~dB})=L_{o}(\mathrm{~dB})+10 \alpha \log \left(d / d_{o}\right)+X_{\sigma}(\mathrm{dB})
$$

Here, $d$ is the distance between transmitter and receiver; $d_{o}$ is the reference distance; $L_{o}$ is the path loss at reference distance $d_{o} ; \alpha$ represents the path loss exponent; and $X_{\sigma}$ is a zero mean Gaussian random variable with standard deviation $\sigma$ that represents the shadowing. The gain factor for the path loss model can be expressed as

$$
L_{d}=\frac{P_{t}}{P_{r}}=L_{o}\left(\frac{d}{d_{o}}\right)^{\alpha} X_{\sigma}
$$

where, $P_{t}$ is the transmit power and $P_{r}$ the received signal power given by $P_{r}(\mathrm{~dB})=P_{t}(\mathrm{~dB})-L_{d}(\mathrm{~dB})$. The channel parameters for in-body and on-body propagation models taken from [21, 23, 24] respectively are listed in Table 6. The signal-to-noise ratio (SNR) at the receiver is expressed as

$$
\gamma(\mathrm{dB})=P_{t}-L_{d}-P_{n}
$$

where $P_{n}$ is the additive white Gaussian noise power and the SNR can be written as $\gamma(\mathrm{dB})=P_{t} /\left(L_{d} P_{n}\right)$.

\subsection{Energy consumption analysis: uncoded transmission}

We assume that a data packet of length $L$ bits is to be transmitted to the hub. In uncoded transmission, data bits are transmitted through the channel without any additional error control overheads. During transmission, PSDU is pre-appended with a physical layer convergence protocol (PLCP) header and a PLCP preamble to create the PPDU. If $M d$ denotes the modulation scheme i.e., $M d \in\{$ BPSK, DBPSK, DQPSK, D8PSK $\}$ with corresponding values for constellation size $M \in\{2,2,4,8\}$ respectively, the time duration to transmit the PPDU for the uncoded system with constellation size $M$ is given by [21]

$$
\begin{aligned}
t_{\mathrm{ON}}^{(U, M d)}=T_{s}\left[N_{\text {preamble }}\right. & +N_{\text {PLCPhdr }} S_{\text {PLCPhdr }} \\
& \left.+\frac{L}{\log _{2} M} S_{\text {PSDU }}\right]
\end{aligned}
$$

Here, $N_{\text {preamble }}$ and $N_{\text {PLCPhdr }}$ denotes the bits in preamble and header of PPDU structure of the narrow band shown in Fig. 2. Quantities $S_{\text {PLCPhdr }}$ and $S_{\text {PSDU }}$ are the spreading factors given in Table 2. For the payload delivered from the MAC layer (i.e., the PSDU), the time duration spent in the transmit state depends on the symbol rate, modulation, and coding schemes. Assuming that the

\begin{tabular}{|c|c|c|c|c|}
\hline Parameter & Value & & & \\
\hline \multirow[t]{2}{*}{ Bandwidth } & \multicolumn{2}{|l|}{ In-body } & \multicolumn{2}{|l|}{$300 \mathrm{KHz}$} \\
\hline & \multicolumn{2}{|l|}{ On-body } & \multicolumn{2}{|l|}{$1 \mathrm{MHz}$} \\
\hline Transmission & \multicolumn{2}{|l|}{ In-body } & \multicolumn{2}{|l|}{$-10 \mathrm{dBm}$} \\
\hline \multirow[t]{3}{*}{ Power } & \multicolumn{2}{|l|}{ On-body } & \multicolumn{2}{|l|}{$-12 \mathrm{dBm}$} \\
\hline & Scenario & On-body NLOS & On-body LOS & In-body \\
\hline & $d_{0}(\mathrm{~cm})$ & 10 & 10 & 5 \\
\hline Channel & $\mathrm{PL}\left(d_{0}\right)(\mathrm{dB})$ & 48.4 & 35.2 & 49.81 \\
\hline Model & $\alpha$ & 5.9 & 3.11 & 4.22 \\
\hline \multirow[t]{2}{*}{ Parameters } & $X_{\sigma}(\mathrm{dB})$ & 5 & 6.1 & 6.81 \\
\hline & $f(\mathrm{GHz})$ & 3.1 & 2.45 & $0.402-0.405$ \\
\hline
\end{tabular}
data packet is successfully received at the receiver and assuming the time taken for reception to be the same as the transmission time, the total energy consumption per

Table 6 Parameters for channel models [21, 23, 24] 


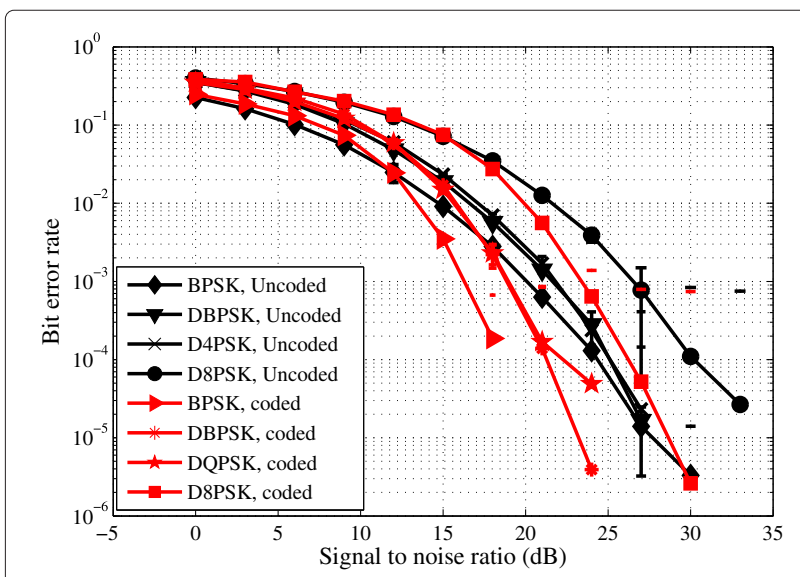

Fig. 2 BER vs SNR for BPSK, DBPSK, DQPSK, and D8PSK schemes: on-body NLOS channel model

bit for the uncoded system for a desired BER performance and employing a modulation scheme $M d$ can be expressed as

$$
\begin{aligned}
E_{\text {Total }}^{(U, \mathrm{Md})}=\frac{1}{L} & {\left[\left((1+\zeta) P_{\mathrm{rf}}^{(U, \mathrm{Md})}+P_{\mathrm{TX} \_ \text {_lec }}\right) t_{\mathrm{ON}}^{(U, \mathrm{Md})}+P_{\mathrm{Wup}} t_{\mathrm{Wup}}\right.} \\
& \left.+P_{\text {Sleep }} t_{\text {Sleep }}+P_{\mathrm{RX}_{-} \text {Elec }} t_{\mathrm{ON}}^{(U, \mathrm{Md})}\right]
\end{aligned}
$$

Here, $P_{\mathrm{rf}}$ is the required transmit power for the desired level of BER, $\zeta$ the power amplifier efficiency given as $\zeta=\frac{\epsilon}{\eta}-1$, where $\epsilon$ is the peak-to-average ratio, and $\eta$ is the drain efficiency (we take $\epsilon$ as 1 and $\eta$ as 0.35 in our analysis [25]); $P_{\mathrm{TX} \_ \text {Elec }}$ and $P_{\mathrm{RX} \_ \text {Elec }}$ respectively represents the circuit power consumption at the transmitter and receiver, $P_{\mathrm{Wup}}$ and $t_{\mathrm{Wup}}$ are the power consumption and duration for the transceiver to wake up from the sleep state, $P_{\text {Sleep }}$ and $t_{\text {Sleep }}$ are the respective values in the sleep state, and $U$ represents an uncoded system. The parameters $P_{\mathrm{TX} \_ \text {Elec }}, P_{\mathrm{RX} \_ \text {Elec }}, P_{\mathrm{Wup}}, t_{\mathrm{Wup}}$, and $P_{\text {Sleep }}$ are specific to the device and $t_{\text {Sleep }}$ depends on the access scheme at the higher layer; $t_{\mathrm{ON}}$ depends on coding as well as modulation scheme and $P_{\mathrm{rf}}$ depends on desired BER, as well as the coding and modulation schemes. Let the RF transmit energy component be denoted as $E_{\mathrm{rf}}^{(U, \mathrm{Md})}=((1+$

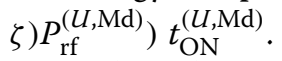

Based on the path loss model, the SNR at the receiver can be expressed in terms of transmission energy as

$$
\gamma^{(U, \mathrm{Md})}=\frac{E_{\mathrm{rf}}^{(U, \mathrm{Md})}}{L_{d} P_{n} t_{\mathrm{ON}}^{(U, \mathrm{Md})}}
$$

where $L_{d}$ is the gain factor for the path loss model at distance separation of $d$ and $P_{n}$ is the total noise power at the receiver. We consider the BPSK modulation scheme as the baseline and compare the energy consumption of DBPSK, DQPSK, and D8PSK modulation schemes. For this, we define SNR loss as the additional SNR in $d B$ required by DBPSK, DQPSK, and D8PSK modulation schemes to achieve the same target BER performance as that of the BPSK for the different WBAN channel models. Hence, SNR loss (SL) for a given target BER can be expressed as

$$
\mathrm{SL}^{(U, \mathrm{Md})}(\mathrm{dB})=\gamma^{(U, \mathrm{Md})}(\mathrm{dB})-\gamma^{(U, \mathrm{BPSK})}(\mathrm{dB})
$$

From (7), for a given target BER, we can express SNR of the differential modulation schemes in terms of SNR of BPSK scheme as $\gamma^{(U, \mathrm{Md})}=\mathrm{SL}^{(U, \mathrm{Md})} \gamma^{(U, \mathrm{BPSK})}$. Hence, we can express the energy consumption of uncoded DBPSK, DQPSK and D8PSK modulation schemes with respect to the uncoded BPSK scheme as

$$
\begin{aligned}
E_{\mathrm{rf}}^{(U, \mathrm{Md})} & =\mathrm{SL}^{(U, \mathrm{Md})} \gamma^{(U, \mathrm{BPSK})} L_{d} P_{n} t_{\mathrm{ON}}^{(U, \mathrm{Md})} \\
& =\mathrm{SL}^{(U, \mathrm{Md})} E_{\mathrm{rf}}^{(U, \mathrm{BPSK})} \frac{t_{\mathrm{ON}}^{(U, \mathrm{Md})}}{t_{\mathrm{ON}}^{(U, \mathrm{BPSK})}}
\end{aligned}
$$

\subsection{Energy consumption analysis: $\mathrm{BCH}$-coded transmission}

The IEEE 802.15.6 standard specifies a systematic $\mathrm{BCH}$ code $(n=63, k=51, t=2)$ for data transmission in the NB PHY. The data packet of length $L$ bits is divided into blocks of $k$ bits and $n-k$ parity check bits are added by the encoder to form $\left\lceil\frac{L}{k}\right\rceil n$ bits and accordingly the transmission time for $\mathrm{BCH}$-coded case can be expressed as

$$
\begin{aligned}
t_{\mathrm{ON}}^{\mathrm{BCH}, \mathrm{Md})}=T_{s}\left[N_{\text {preamble }}\right. & +N_{\text {PLCPhdr }} S_{\text {PLCPhdr }} \\
& \left.+\frac{\left\lceil\frac{L}{k}\right\rceil n}{\log _{2} M} S_{\text {PSDU }}\right]
\end{aligned}
$$

When compared to the uncoded case, the time duration for data packet transmission is increased due to the additional parity check bits. The SNR at the receiver with $\mathrm{BCH}$-coded transmission can be expressed as

$$
\gamma^{(\mathrm{BCH}, \mathrm{Md})}=\frac{E_{\mathrm{rf}}^{(\mathrm{BCH}, \mathrm{Md})}}{L_{d} P_{n} t_{\mathrm{ON}}^{(\mathrm{BCH}, \mathrm{Md})}}
$$

Employing $\mathrm{BCH}$ codes results in coding gain, which is defined as the additional SNR that would be required for an uncoded system to provide the same level of BER performance as that of the coded system for a given modulation scheme and can be expressed as

$$
\mathrm{CG}^{(\mathrm{BCH}, \mathrm{Md})}(\mathrm{dB})=\gamma^{(U, \mathrm{Md})}(\mathrm{dB})-\gamma^{(\mathrm{BCH}, \mathrm{Md})}(\mathrm{dB}
$$

Notice that (11) is computed for a given target BER requirement. Considering performance of uncoded system as the baseline, we evaluate coding gains at three 
different BER values: $10^{-3}, 10^{-4}$, and $10^{-5}$. For a given $\mathrm{BER}$ requirement, the received SNR with $\mathrm{BCH}$ coding can be expressed in terms of SNR requirement of uncoded transmission as

$$
\gamma^{(\mathrm{BCH}, \mathrm{Md})}=\gamma^{(U, \mathrm{Md})} / \mathrm{CG}^{(\mathrm{BCH}, \mathrm{Md})}
$$

Combining (6) and (12), for a target BER requirement, we can express SNR for BCH-coded case, $\gamma^{(\mathrm{BCH}, \mathrm{Md})}$ as

$$
\gamma^{(\mathrm{BCH}, \mathrm{Md})}=\frac{E_{\mathrm{rf}}^{(U, \mathrm{Md})}}{L_{d} P_{n} \mathrm{CG}^{(\mathrm{BCH}, \mathrm{Md})} t_{\mathrm{ON}}^{(U, \mathrm{Md})}}
$$

It may be noted that energy consumption reduction is achievable for positive values of coding gain (expressed in $\mathrm{dB}$ ). This benefit is achieved along with reduction of effective data rate or with increase of band width requirement. From (8), (10), and (13), we can express the transmission energy requirement for $\mathrm{BCH}$-coded system in terms of transmission energy requirement of uncoded BPSK system as

$$
E_{\mathrm{rf}}^{(\mathrm{BCH}, \mathrm{Md})}=\frac{\mathrm{SL}^{(U, \mathrm{Md})} E_{\mathrm{rf}}^{(U, \mathrm{BPSK})} t^{(\mathrm{BCH}, \mathrm{Md})}}{\mathrm{CG}^{(\mathrm{BCH}, \mathrm{Md})} t_{\mathrm{ON}}^{(U, \mathrm{BPSK})}}
$$

Here, $E_{\mathrm{rf}}^{(\mathrm{BCH}, \mathrm{Md})}$ represents the total transmit energy consumed by a bio-sensor node when $\mathrm{BCH}$ coding is employed, for a given BER requirement. The above equation shows that the energy consumption of a sensor node increases due to the overheads introduced by the error control coding and strongly depends on higher order modulation schemes. At the same time, a reduction in energy consumption is achieved as a result of coding gain provided by the error control coding scheme. Whenever this reduction in energy consumption due to coding gain outweighs the increased energy expenditure due to the transmission of overhead as well as additional energy consumption owing to the use of higher constellation size, a net benefit in terms of energy consumption is possible over the BPSK scheme. However, we need to consider the additional energy consumed for decoding the data frame. The decoding energy at the hub is the total energy required for decoding $\left\lceil\frac{L}{k}\right\rceil$ blocks. The energy to decode one block, $E_{\mathrm{dec}}$, is given by [16]:

$$
E_{\mathrm{dec}}=\left(2 n t+2 t^{2}\right)\left(E_{\mathrm{add}}+E_{\text {mult }}\right),
$$

where $E_{\text {add }}$ and $E_{\text {mult }}$ respectively denote the energy consumptions involved in addition and multiplication of field elements in $\operatorname{GF}\left(2^{m}\right)[16]$. Here, $t$ is the error correcting capability of binary $\mathrm{BCH}$ code of length $n$ and $m=$ $\left\lfloor\log _{2} n+1\right\rfloor$. The energy consumption is

$$
E_{D e c}^{\mathrm{BCH}}=E_{\mathrm{dec}}\left\lceil\frac{L}{k}\right\rceil
$$

We assume the energy consumed for encoding to be negligible [16]. Hence, when $\mathrm{BCH}$ coding is employed, assuming successful reception of data, our goal is to minimize the quantity given below:

$$
\begin{aligned}
E_{\mathrm{bit}}=\frac{1}{L} & {\left[\left((1+\zeta) P_{\mathrm{rf}}^{(\mathrm{BCH}, \mathrm{Md})}+P_{\mathrm{TX} \_ \text {Elec }}\right) t_{\mathrm{ON}}^{(\mathrm{BCH}, \mathrm{Md})}+P_{\mathrm{Wup}} t_{\mathrm{Wup}}\right.} \\
& \left.+P_{\text {Sleep }} t_{\text {Sleep }}+E_{\mathrm{dec}}\left\lceil\frac{L}{k}\right\rceil+P_{\mathrm{RX} \_ \text {Elec }} t_{\mathrm{ON}}^{(\mathrm{BCH}, \mathrm{Md})}\right]
\end{aligned}
$$

It may be noted that the objective function given by (17) represents the total energy consumed by the WBAN sensor node as well as the hub for successful transmission of one bit of information and is a function of the constellation size of the modulation scheme employed, and SNR required for a given BER performance, that depends on the WBAN channel model as well as the coding scheme employed. In Section 3, we find the energy consumption for various modulation schemes and for various code rates.

\section{Results and discussions}

In this section, we evaluate the energy consumed by various modulation schemes in the context of WBAN narrow band physical layer. We perform extensive Monte Carlo simulations for a WBAN with star topology in which the node transfers data directly to the hub. We assume a contention-free MAC scheme such as sched-

\begin{tabular}{|c|c|c|}
\hline Parameter & Nordic nRF24L01+ & zarlink zl70101 \\
\hline Supply voltage & $3 \mathrm{~V}$ & $3 \mathrm{~V}$ \\
\hline Transmit current $\left(/_{T X}\right)$ & $7.5 \mathrm{~mA}$ & $5 \mathrm{~mA}$ \\
\hline Receive current $\left(I_{R X}\right)$ & $13.1 \mathrm{~mA}$ & $5 \mathrm{~mA}$ \\
\hline Sleep current (/sleep) & $900 \mathrm{nA}$ & $250 \mathrm{nA}$ \\
\hline Idle current (lidle $)$ & $26 \mu \mathrm{A}$ & $1 \mathrm{~mA}$ \\
\hline Wakeup current (/Wup); 1.5 ms twup & $400 \mu \mathrm{A}$ & - \\
\hline Wakeup current (/Wup); 1.15 s twup & - & $250 \mathrm{nA}$ \\
\hline Transmit circuit energy/bit $E_{T X_{2} E l e c}$ & $11.25 \mathrm{~nJ} / \mathrm{bit}$ & $18.75 \mathrm{~nJ} / \mathrm{bit}$ \\
\hline Receive circuit energy/bit $E_{\mathrm{RX} \_E l e c}$ & $11.25 \mathrm{~nJ} / \mathrm{bit}$ & $18.75 \mathrm{~nJ} / \mathrm{bit}$ \\
\hline$E_{\text {add }}(\mathrm{W} / \mathrm{Hz})$ & \multicolumn{2}{|c|}{$3.3 \times 10^{-14} \mathrm{~m}$} \\
\hline$E_{\text {mult }}(\mathrm{W} / \mathrm{Hz})$ & \multicolumn{2}{|c|}{$3.7 \times 10^{-14} \mathrm{~m}^{3}$} \\
\hline Power amplifier efficiency & \multicolumn{2}{|l|}{1.85} \\
\hline
\end{tabular}
uled uplink/downlink access mechanism. We consider different propagation models: in-body and on-body (both LOS as well as non-LOS). The parameters of channel model and the electrical parameters of the transceiver chips are given in Tables 6 and 7, respectively. First of

Table 7 Transceiver electrical specifications $[26,27]$ 
all, we group bits into data packet of length $L$ which is divided into blocks of $k$ bits and $(n-k)$ parity bits are appended to each block (i.e., $n=63, k=51, t=2$ ). The encoded bits are modulated using the constellation mapping given in Tables 3,4 , and 5 . We then introduce distance-dependent path loss, lognormal shadowing, and additive white Gaussian noise into the simulations. The following modulation schemes are considered: BPSK, DBPSK, DQPSK, D8PSK, NC2FSK, NC4FSK, NC8FSK. When we go for higher order modulation schemes, the increase in complexity is higher at the receiver than at the transmitter side since the receiver has to make more comparisons to apply the minimum distance criterion. However, we do not consider the additional energy consumed in this process. The BER is determined by transmitting as many as $5 \times 10^{6}$ blocks of 51 bits each. The BER evaluation process is then repeated 10,000 times with a different seed value for channel noise. The average BER is calculated from these 10,000 sample values. The coding gain values are evaluated for different target BER values $\left(10^{-3}, 10^{-4}, 10^{-5}\right)$. In the graphs, simulation results are plotted with $95 \%$ confidence intervals. The energy calculations are done by taking the energy consumption for uncoded NLOS at a desired BER of $10^{-3}$ for a transmit power of $-42 \mathrm{~dB}$ as a baseline (this corresponds to SNR value of $20.7 \mathrm{~dB}$ at a distance separation of $20.9 \mathrm{~cm}$ between node and hub when the noise power is $-130 \mathrm{~dB}$ and for the NLOS parameters given in Table 6). The power amplifier efficiency and PAPR values for computing the total RF power are based on [25]. Further, the electrical parameters of Table 7 are used for computing the energy consumption of circuit electronics and power in different operating states of the node, based on Zarlink ZL70101 [26] and Nordic nRF24L01+ [27] transceivers, respectively. On-body communication takes place in the $2.4-\mathrm{GHz}$ industrial, scientific, and medical band (for which we select the parameters of the 2.4-GHz transceiver nRF24L01+), which support higher data rates than in-body communication in the 402-405-MHz MICS band (for which we select the parameters of the the implantable transceiver ZL70101). The SNR loss and coding gains for different target BER values are evaluated to determine the energy consumption of various modulation schemes for the desired error performance.

Figures 2, 3, and 4 show the BER vs SNR plots for coded as well as the uncoded case for on-body NLOS, LOS, and in-body propagation models, respectively. The BER curve has been plotted until the bit error of the order of $10^{-6}$. The BPSK system is taken as the reference and the performance of differential modulation schemes with constellation sizes of 2, 4, and 8 specified in IEEE 802.15.6 are evaluated. The bit stream is mapped to a corresponding complex-valued sequence according

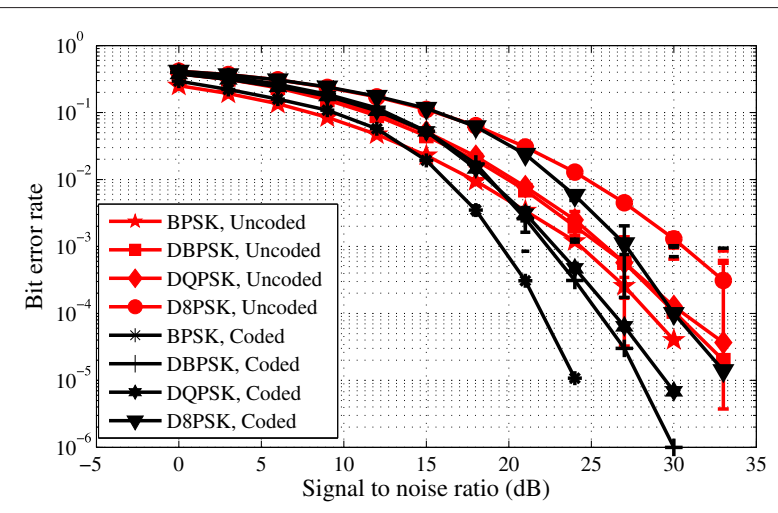

Fig. 3 BER vs SNR for BPSK, DBPSK, DQPSK, and D8PSK schemes: on-body LOS channel model

to constellation mapping given in Tables 3,4 , and 5. It is observed that in-body communication has higher BER as compared to the on-body scenario, owing to the considerable path loss experienced by the in-body channel. The BER performance of on-body non-LOS communication is observed to be better than that of on-body LOS model owing to higher shadow fading standard deviation experienced by the on-body LOS propagation model. It may be noted that DBPSK, DQPSK, and D8PSK modulation schemes require higher SNR for a desired BER performance as compared to BPSK. This difference in SNR requirement has been defined as SNR loss in Section 2. Further, the use of $\mathrm{BCH}$ coding reduces the required SNR for a target BER performance and the corresponding SNR gain is defined as coding gain in Section 2. The values for SNR loss and coding gain are summarized in Tables 8 and 9 respectively for various propagation models and various values of target BER. From Table 8, the SNR loss is observed to be higher for the higher order modulation schemes. This is because as

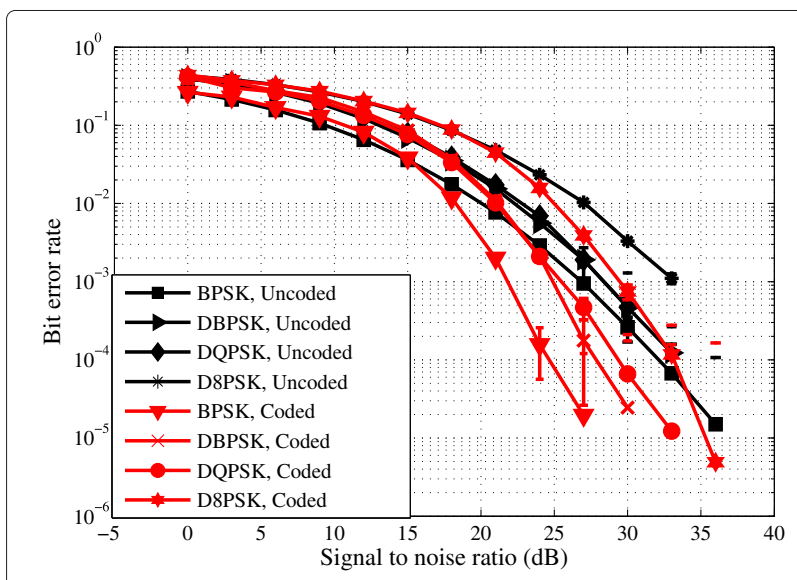

Fig. 4 BER vs SNR for BPSK, DBPSK, DQPSK, and D8PSK schemes: in-body channel model 
Table 8 SNR loss of DBPSK, DQPSK, and D8PSK

\begin{tabular}{|c|c|c|c|c|c|c|c|c|c|c|}
\hline \multirow[b]{2}{*}{$\begin{array}{l}\text { Channel } \\
\text { model }\end{array}$} & \multirow{2}{*}{$\begin{array}{l}\text { BER: } \\
\text { Modulation }\end{array}$} & \multicolumn{3}{|c|}{$10^{-3}$} & \multicolumn{3}{|c|}{$10^{-4}$} & \multicolumn{3}{|c|}{$10^{-5}$} \\
\hline & & SNR & $\begin{array}{l}\text { SNR } \\
\text { (BPSK) }\end{array}$ & SL & SNR & $\begin{array}{l}\text { SNR } \\
\text { (BPSK) }\end{array}$ & SL & SNR & $\begin{array}{l}\text { SNR } \\
\text { (BPSK) }\end{array}$ & SL \\
\hline On-body & DBPSK & 21.7 & & 1 & 25.5 & & 1.3 & 27.76 & & 0.21 \\
\hline \multirow[t]{3}{*}{ NLOS } & DQPSK & 21.95 & 20.7 & 1.25 & 25.65 & 24.2 & 1.45 & 28.6 & 27.55 & 1.05 \\
\hline & D8PSK & 26.68 & & 5.98 & 30.25 & & 6.05 & 33.2 & & 5.65 \\
\hline & DBPSK & 25.6 & & 1.35 & 30.0 & & 2.3 & 33.1 & & 1.1 \\
\hline On-body & DQPSK & 26.3 & 24.25 & 2.05 & 30.27 & 27.7 & 2.57 & 33.70 & 32 & 1.7 \\
\hline \multirow[t]{2}{*}{ LOS } & D8PSK & 30.27 & & 6.02 & 34.45 & & 6.75 & 38.9 & & 6.9 \\
\hline & DBPSK & 28.28 & & 1.68 & 33.62 & & 2.27 & 37.3 & & 1.15 \\
\hline \multirow[t]{2}{*}{ In-body } & DQPSK & 29.5 & 26.6 & 2.9 & 34.0 & 31.35 & 2.65 & 37.9 & 36.15 & 1.75 \\
\hline & D8PSK & 33.24 & & 6.64 & 38.3 & & 6.95 & 43.25 & & 7.1 \\
\hline
\end{tabular}

given in Tables 3, 4, and 5, the symbols are closer to each other for the higher order modulation schemes compared to lower order modulation schemes and higher transmit power is required for the higher order modulation schemes to achieve a desired error performance. Further, maximum SNR loss is observed for the in-body channel model. Table 9 summarizes the coding gain values determined for the different channel models and for different BER values $\left(10^{-3}, 10^{-4}, 10^{-5}\right)$. Recall that the IEEE 802.15.6 standard specifies the use of $\mathrm{BCH}(63,51,2)$ code and the use of DBPSK, DQPSK, and D8PSK modulation schemes for data transmission at different rates in the NB PHY [5]. The coding gain is highest for BPSK scheme compared to the differential modulation schemes. Among the differential schemes, it is observed that the coding gain achievable is maximum if D8PSK modulation is employed.
Next, we plot the transmission time required for the different modulation schemes based on the narrow band physical layer parameters. The transmission time is evaluated for the case when a data packet of size 1000 bits is transferred from the node to the hub. Figure 5 shows the time required for the uncoded as well the coded cases for on-body NLOS communication and in-body communication scenarios. The use of higher order modulation schemes reduces the transmission time considerably. The in-body communication operates at a lower bit rate; whereas the on-body communication takes place at a higher bit rate [5]. Thus, on-body communication has a lower transmission time. The in-body communication has a transmission time of $7.5 \mathrm{~ms}$ when $\mathrm{BCH}$ coding $(63,51)$ specified in IEEE802.15.6 and DBPSK modulation scheme is used. The transmission time reduces to $3.1 \mathrm{~ms}$ for D8PSK scheme, and

Table 9 Coding gain at code rate specified in IEEE 802.15.6

\begin{tabular}{|c|c|c|c|c|c|c|c|c|c|c|}
\hline \multirow[b]{2}{*}{$\begin{array}{l}\text { Channel } \\
\text { model }\end{array}$} & \multirow{2}{*}{$\begin{array}{l}\text { BER } \\
\text { Modulation }\end{array}$} & \multicolumn{3}{|c|}{$10^{-3}$} & \multicolumn{3}{|c|}{$10^{-4}$} & \multicolumn{3}{|c|}{$10^{-5}$} \\
\hline & & $\begin{array}{l}\text { SNR } \\
\text { uncoded }\end{array}$ & $\begin{array}{l}\text { SNR } \\
\text { coded }\end{array}$ & $\begin{array}{l}\text { Coding } \\
\text { gain }\end{array}$ & $\begin{array}{l}\text { SNR } \\
\text { uncoded }\end{array}$ & $\begin{array}{l}\text { SNR } \\
\text { coded }\end{array}$ & $\begin{array}{l}\text { Coding } \\
\text { gain }\end{array}$ & $\begin{array}{l}\text { SNR } \\
\text { uncoded }\end{array}$ & $\begin{array}{l}\text { SNR } \\
\text { coded }\end{array}$ & $\begin{array}{l}\text { Coding } \\
\text { gain }\end{array}$ \\
\hline & BPSK & 20.7 & 16.5 & 4.2 & 24.2 & 18.6 & 5.6 & 27.55 & 20.29 & 7.26 \\
\hline On-body & DBPSK & 21.7 & 18.96 & 2.74 & 25.5 & 21.7 & 3.8 & 27.76 & 23.54 & 4.22 \\
\hline \multirow[t]{3}{*}{ NLOS } & DQPSK & 21.95 & 19.05 & 2.90 & 25.65 & 21.71 & 3.94 & 28.6 & 24.2 & 4.4 \\
\hline & D8PSK & 26.68 & 23.4 & 3.28 & 30.25 & 26.1 & 4.15 & 33.2 & 28 & 5.2 \\
\hline & BPSK & 24.25 & 19.7 & 4.55 & 27.7 & 22.25 & 5.45 & 32 & 23.95 & 8.05 \\
\hline On-body & DBPSK & 25.6 & 22.665 & 2.935 & 30.0 & 25.58 & 4.42 & 33.1 & 28.5 & 4.6 \\
\hline \multirow[t]{4}{*}{ LOS } & DQPSK & 26.3 & 22.9 & 3.4 & 30.27 & 26.6 & 4.47 & 33.70 & 29.05 & 4.65 \\
\hline & D8PSK & 30.27 & 26.8 & 3.47 & 34.45 & 29.9 & 4.55 & 38.9 & 33.19 & 5.71 \\
\hline & BPSK & 26.6 & 21.8 & 4.8 & 31.35 & 24.4 & 6.95 & 36.15 & 27.25 & 8.9 \\
\hline & DBPSK & 28.28 & 24.70 & 3.58 & 33.62 & 28.3 & 5.32 & 37.3 & 29.11 & 5.49 \\
\hline \multirow[t]{2}{*}{ In-body } & DQPSK & 29.5 & 25.55 & 3.95 & 34 & 28.5 & 5.5 & 37.9 & 32.27 & 5.63 \\
\hline & D8PSK & 33.24 & 29.1 & 4.14 & 38.3 & 32.65 & 5.65 & 43.25 & 36.9 & 6.35 \\
\hline
\end{tabular}

The italicized data specifies the coding gain 


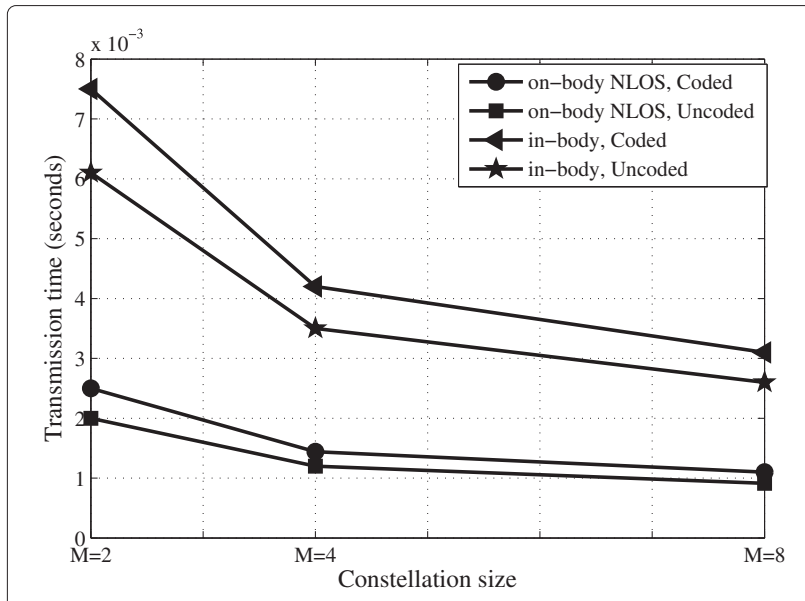

Fig. 5 Transmission time for uncoded and $\mathrm{BCH}$-coded schemes for transferring a data packet of 1000 bits: on-body NLOS and in-body communication

for uncoded D8PSK scheme, the transmission time is $2.6 \mathrm{~ms}$.

Figures 6 and 7 show the total energy consumption per bit for the different modulation schemes for target BER of $10^{-4}$ and $10^{-5}$, respectively. It is observed that the total energy consumption for higher order modulation schemes specified in the standard, i.e., DQPSK and D8PSK, are lower. This can be explained as follows: when higher order modulation schemes are used, the transmission time given by (4) and (9) is reduced by a factor $\log _{2} M$. This reduces the circuit energy consumption at the transmitter as well as at the receiver. The presence of coding gain reduces the RF power requirement as given by (14). As the order of modulation scheme is increased, the reduction of transmission time has dominant effect causing higher order modulation

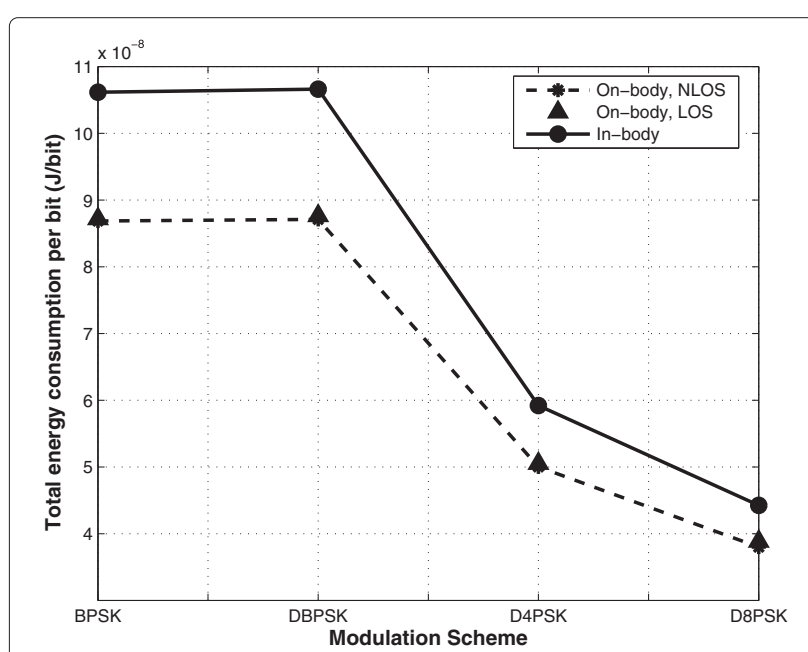

Fig. 6 Total energy consumption per bit (J/b) for BPSK, DBPSK, DQPSK, and D8PSK schemes with BCH Coding: at target BER of $10^{-4}$

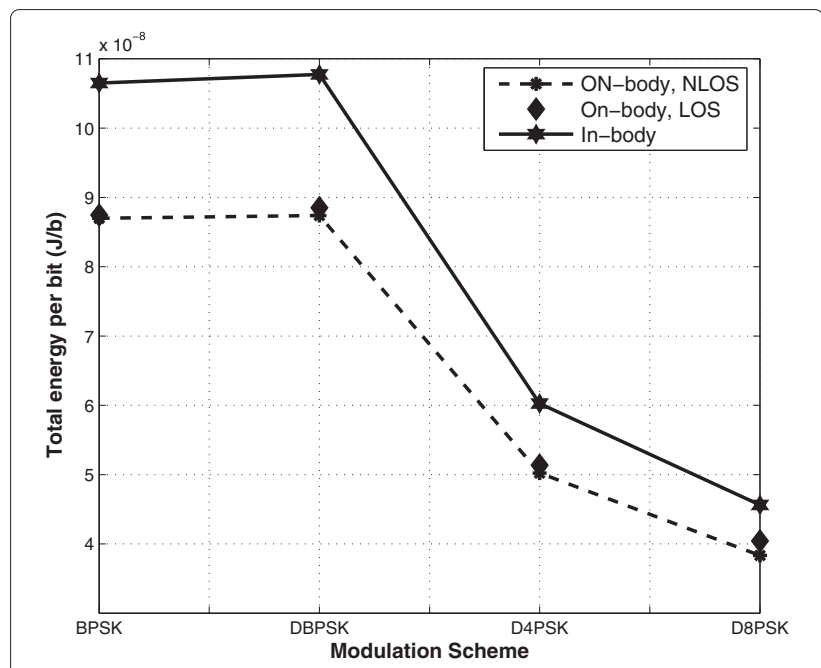

Fig. 7 Total energy consumption per bit (J/b) for BPSK, DBPSK, DQPSK, and D8PSK schemes with BCH Coding: at target BER of $10^{-5}$

schemes to exhibit lower energy consumption as compared to that incurred when lower order modulation schemes are used. However, it may be noted that as the constellation size increases, the complexity of the modulation and demodulation algorithms at the nodes increases.

Figure 8 shows the total energy consumption per bit when different code rates $(\mathrm{k} / \mathrm{n})$ are used for $\mathrm{BCH}$ encoding. We consider $\mathrm{BCH}$ codes with different specifications, i.e., $\mathrm{BCH}(127,43,14), \mathrm{BCH}(127,64,10), \mathrm{BCH}$ $(63,39,4), \mathrm{BCH}(63,45,3)$, and $\mathrm{BCH}(63,51,2)$ [28]. It is observed that for all the code rates considered, higher order modulation results in lower power consumption. The coding gain determined for different code rates for NLOS channel model for a desired error rate of $10^{-4}$ is summarized in Table 10. Results show that, for a given $\mathrm{BER}$, when the code rate $(k / n)$ increases, the coding gain reduces. Further, from Table 10, it is observed that for

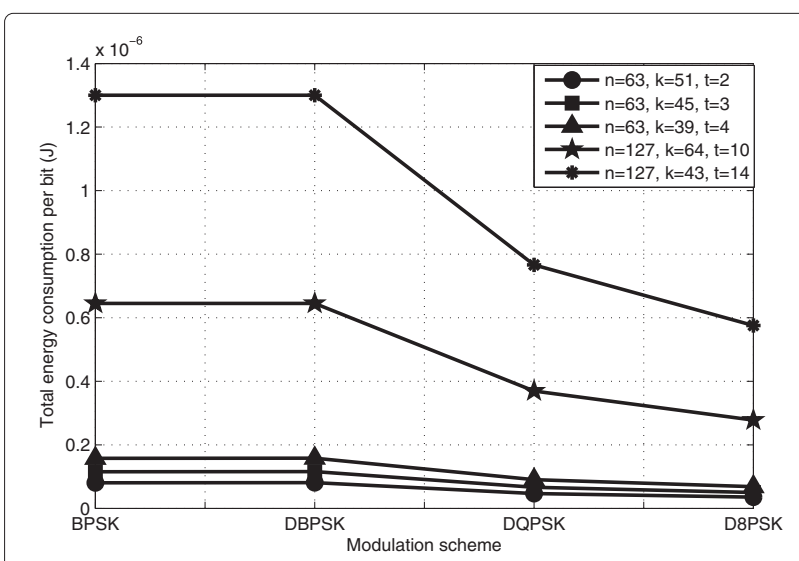

Fig. 8 Total energy consumption per bit (J/b) of modulation schemes when code rate is varied: BER $10^{-4}$, NLOS channel model 
Table 10 Coding gain for different code rates: NLOS channel model, BER $=10^{-4}$

\begin{tabular}{|c|c|c|c|c|c|c|c|c|c|}
\hline \multirow[b]{2}{*}{$\begin{array}{l}\text { Code rate } \\
(n, k, t)\end{array}$} & \multirow{2}{*}{$\begin{array}{l}\text { Modulation: } \\
\text { SNR } \\
\text { Coded }\end{array}$} & \multicolumn{2}{|c|}{ DBPSK } & \multicolumn{3}{|c|}{ DQPSK } & \multicolumn{3}{|c|}{ D8PSK } \\
\hline & & $\begin{array}{l}\text { SNR } \\
\text { uncoded }\end{array}$ & $\begin{array}{l}\text { Coding } \\
\text { gain(dB) }\end{array}$ & $\begin{array}{l}\text { SNR } \\
\text { coded }\end{array}$ & $\begin{array}{l}\text { SNR } \\
\text { uncoded }\end{array}$ & $\begin{array}{l}\text { Coding } \\
\text { gain }(\mathrm{dB})\end{array}$ & $\begin{array}{l}\text { SNR } \\
\text { coded }\end{array}$ & $\begin{array}{l}\text { SNR } \\
\text { uncoded }\end{array}$ & $\begin{array}{l}\text { Coding } \\
\text { gain }(d B)\end{array}$ \\
\hline $63,51,2$ & 21.7 & & 3.8 & 21.71 & & 3.94 & 26.1 & & 4.15 \\
\hline $63,45,3$ & 21.3 & & 4.2 & 21.1 & & 4.55 & 25.1 & & 5.15 \\
\hline $63,39,4$ & 19.87 & 25.5 & 5.63 & 18.85 & 25.65 & 6.8 & 23.2 & 30.25 & 7.05 \\
\hline $127,64,10$ & 18.05 & & 7.45 & 15.75 & & 9.9 & 20 & & 10.25 \\
\hline $127,43,14$ & 17.95 & & 7.55 & 14.275 & & 11.375 & 18.28 & & 11.97 \\
\hline
\end{tabular}

The italicized data specifies the coding gain

a desired error performance at a given code rate, the coding gain is maximum for higher order modulation scheme.

Figure 9 shows the error performance of uncoded NCMFSK and uncoded differential MPSK schemes for values of $M=4$ and $M=8$, for on-body LOS channel model. We also plot the error performance of uncoded BPSK as well, which is considered as the baseline modulation in this paper. It is observed that the performance of differential MPSK modulation scheme degrades as the value of $M$ increases. For a desired error rate of $10^{-3}$, DQPSK and D8PSK require additional 2.05 and $6.02 \mathrm{~dB}$ respectively over the uncoded BPSK scheme. Whereas the NC-MFSK scheme requires 9.5 and $12.8 \mathrm{~dB}$ less than that required for BPSK for the same error performance. Hence, the transmission power required for NC-MFSK for a desired error rate is lower compared to all the modulation schemes specified in IEEE 802.15.6.

Figure 10 shows the total energy consumption per bit of uncoded NC-MFSK and uncoded differential MPSK schemes for different constellation sizes for on-body NLOS channel model model for a desired BER of $10^{-4}$. Owing to the lower transmission power requirement of

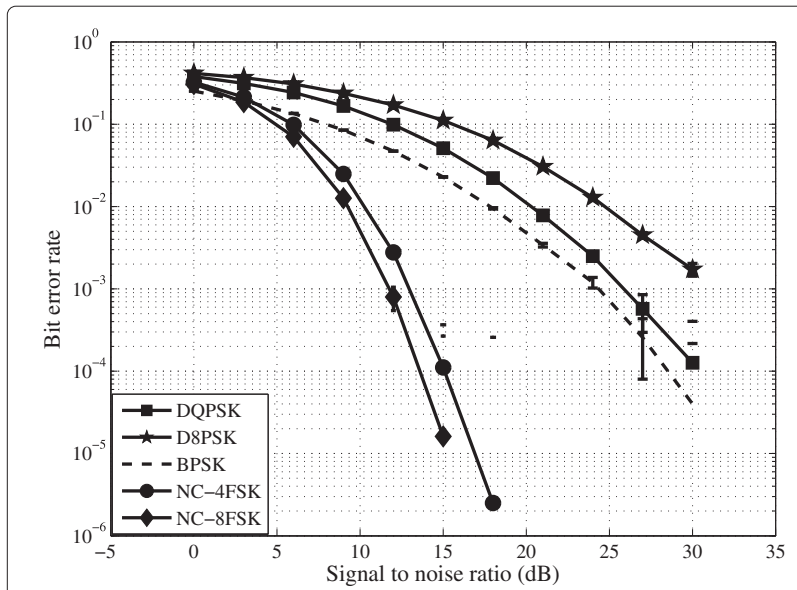

Fig. 9 Error performance of NC-MFSK and differential MPSK schemes: LOS channel model
NC-MFSK scheme, the total energy consumption is lower compared to the differential MPSK schemes.

Next, we consider the impact of modulation scheme on reliable hop distance. Figure 11 shows the reliability (expressed in terms of packet acceptance rate:PAR) vs hop distance for NLOS channel model for uncoded transmission. Here, we fix the transmit power and vary the node-to-hub hop length. It is observed that the hop distance for reliable communication is reduced when higher order differential MPSK modulation schemes are used. For example, when D8PSK modulation is employed, for a PAR of $90 \%$, the reliable hop distance is reduced by approximately $3 \mathrm{~cm}$ compared to the lower order differential modulation schemes. Hence, the higher order modulation scheme requires higher transmit power for a desired error performance. As mentioned earlier, this increase in power consumption is offset by the reduced transmission time. However, when NC-MFSK modulation schemes are employed, we observe significant improvement in reliable hop distance. The NC-MFSK modulation with values $\mathrm{M}=2,4$ and 8 extends the reliable hop distance by $6 \mathrm{~cm}, 10 \mathrm{~cm}$ and $12 \mathrm{~cm}$ respectively over the BPSK scheme.

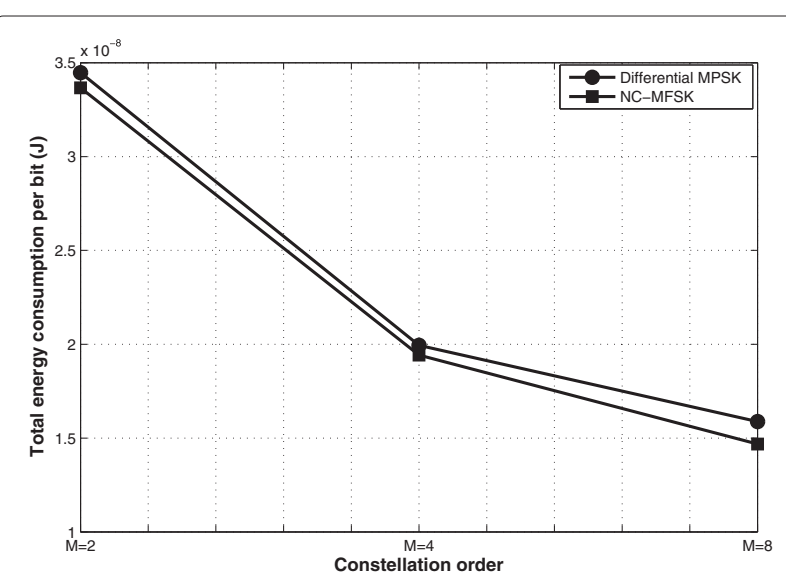

Fig. 10 Total energy consumption per bit for NC-MFSK and differential MPSK schemes: NLOS channel model , $B E R=10^{-4}$ 


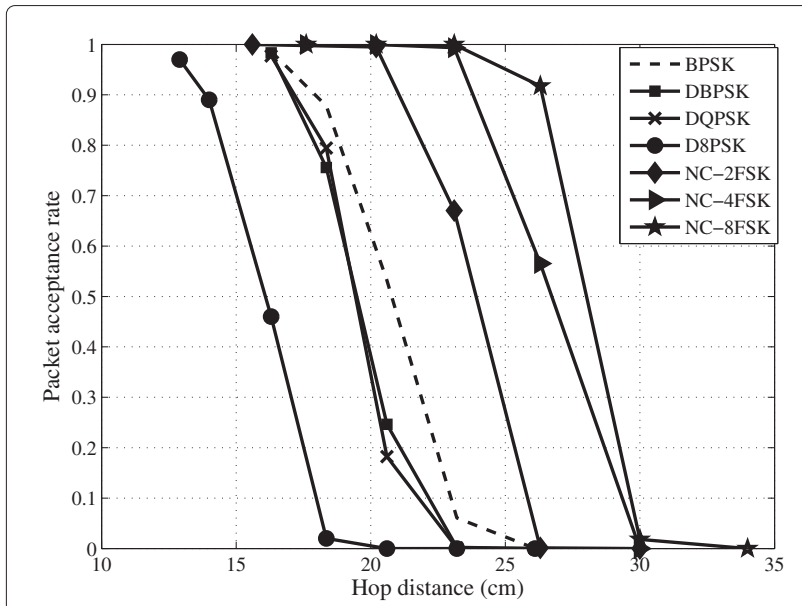

Fig. 11 Packet acceptance rate vs hop distance for NC-MFSK and differential MPSK schemes: NLOS channel model

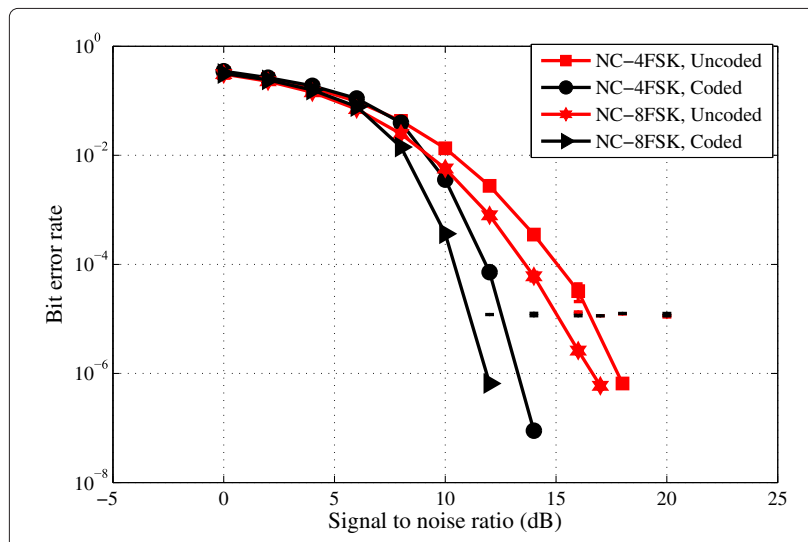

Fig. 12 Bit error rate vs SNR for NC-MFSK-coded and uncoded schemes: LOS channel model

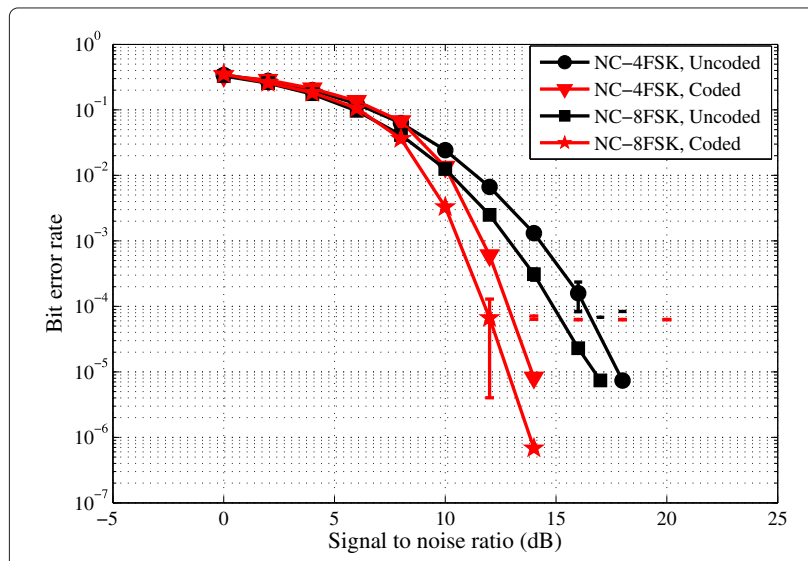

Fig. 13 Bit error rate vs SNR for NC-MFSK-coded and uncoded schemes: NLOS channel model

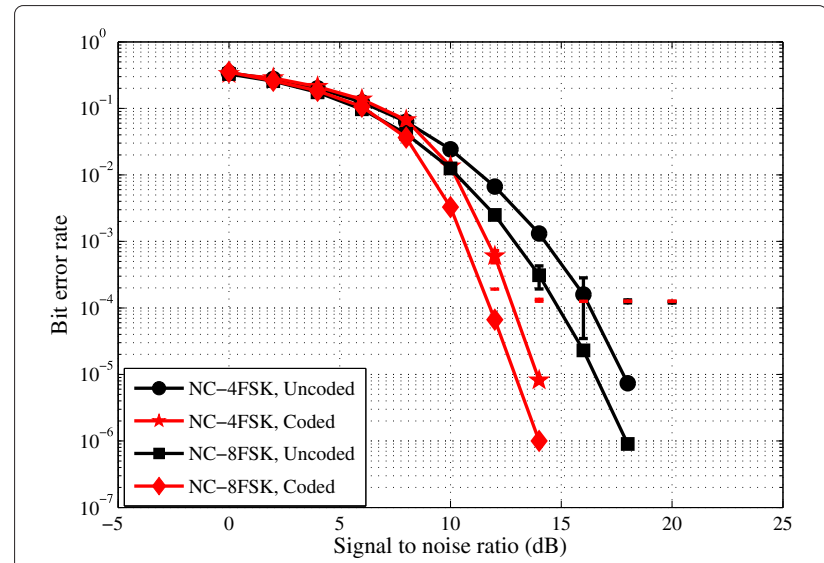

Fig. 14 Bit error rate vs SNR for NC-MFSK-coded and uncoded schemes: in-body channel model

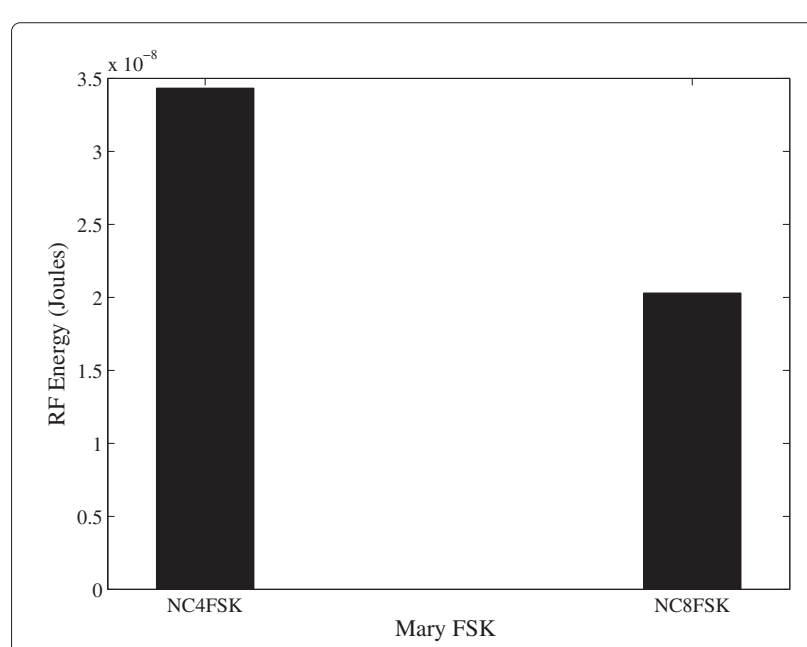

Fig. 15 Transmit energy requirement for $M=4$ and $M=8$ for $M$-ary FSK

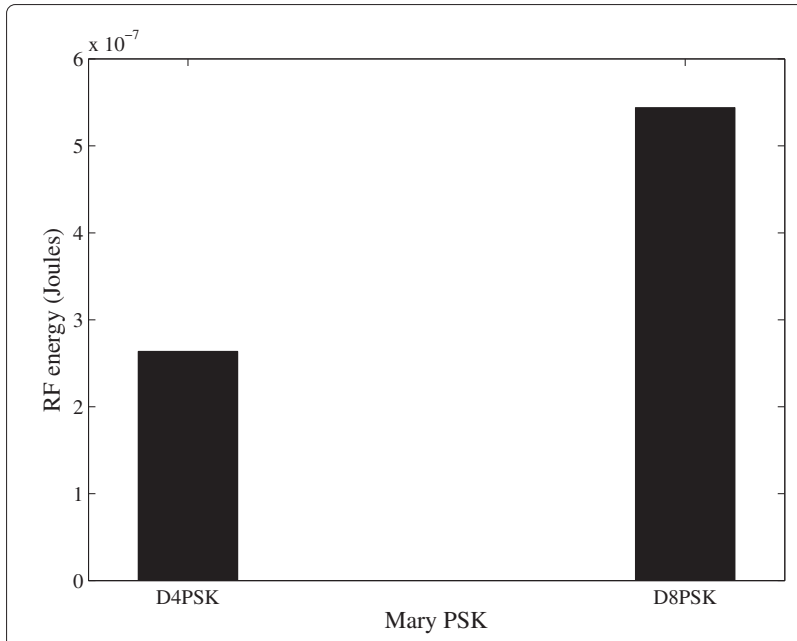

Fig. 16 Transmit energy requirement for $M=4$ and $M=8$ for $M$-ary PSK 
Next, we present additional results for the coded NCMFSK sheme. Figures 12, 13, and 14 show the BER vs SNR plots for the on-body LOS, on-body NLOS, and the in-body communications, respectively. It is observed that the NC-8FSK scheme performs significantly better than NC-4FSK scheme in terms of BER. A comparison of the constellations of $M$-ary orthogonal schemes such as 8 FSK and 4FSK reveals that the distance between message points of 8FSK is larger than the distance between message points of 4FSK, for a given peak transmission power. Accordingly in the AWGN channel, 8FSK outperforms 4FSK in terms of BER. Figures 15 and 16 respectively show the transmit energy requirements for NC-MFSK and $M$ ary differential PSK schemes for meeting a target BER criterion of $10^{-4}$. Results show that NC-8FSK has the minimum transmit energy requirement among all the four schemes considered. Figure 17 compares the total energy consumption per bit of differential MPSK scheme and NC-MFSK with $\mathrm{BCH}$ coding. The results establish that the NC-MFSK outperforms differential MPSK in terms of total energy requirement. Figure 18 shows the improvement in reliable hop distance for the coded and uncoded NC-MFSK scheme. In-body communication scenario is considered. The results establish that PAR is higher for coded NC-MFSK scheme. This leads to reliable hopdistance extention.

\section{Conclusions}

In this paper, we investigated the performance of various modulation schemes specified in the IEEE 802.15.6 WBAN standard in terms of energy consumed by the node for successfully transferring a packet to the hub. An analytical model was presented to compute the energy consumed. The error rate performance of various modulation schemes were also evaluated. Our results showed that, when higher order differential schemes

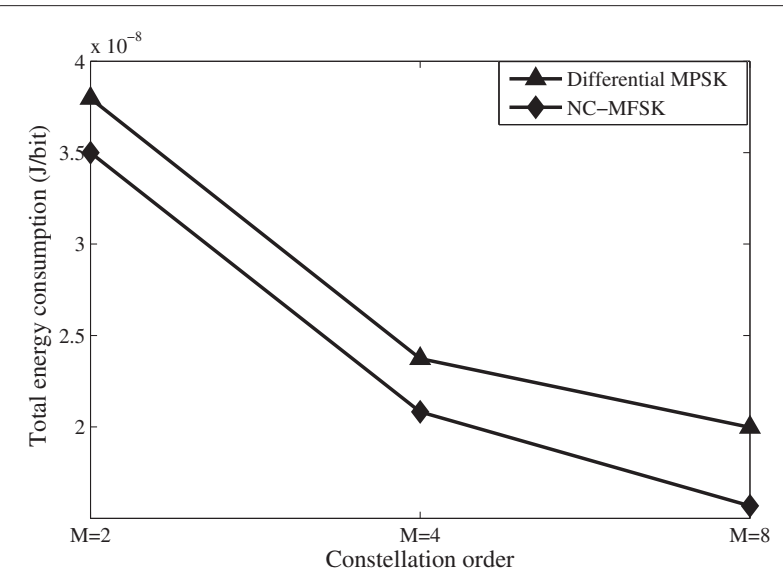

Fig. 17 Total energy consumption for MPSK and NC-FSK schemes when $\mathrm{BCH}$ coding is used

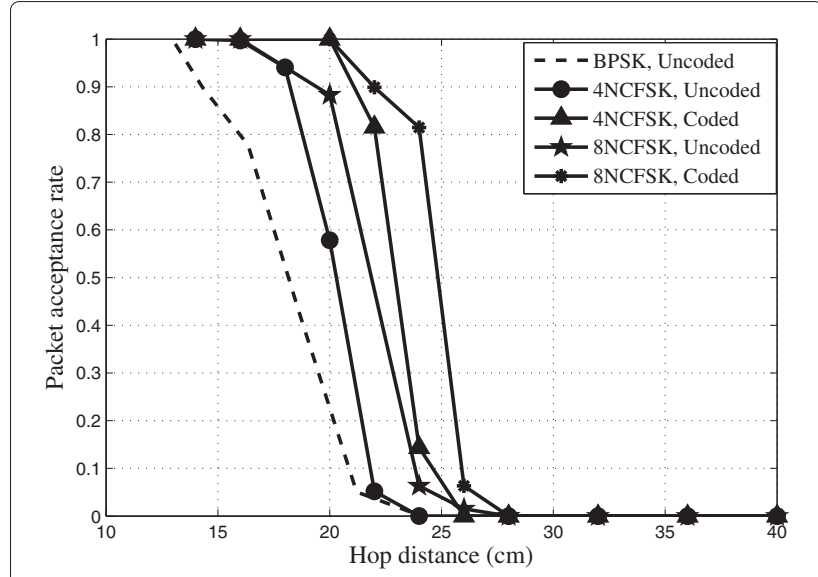

Fig. 18 Reliable hop distance improvement when $\mathrm{BCH}$-coded NC-FSK is used in in-body communication scenario

specified by IEEE 802.15.6 are employed in WBAN, the total energy consumption involved in successful transmission of a packet is reduced significantly. The total energy consumption remains lower for higher order modulation schemes even when $\mathrm{BCH}$ codes with various code rate specifications are employed. We also establish that use of non-coherent MFSK as an alternate modulation scheme can further reduce the energy consumption.

\section{Authors' contributions}

The authors (KSD and AVB) have equally contributed to the contents/work presented in this paper. Both authors read and approved the final manuscript.

\section{Competing interests}

The authors declare that they have no competing interests.

\section{Author details}

${ }^{1}$ Research scholar, Department of Electronics and Communication Engineering, National Institute of Technology, Calicut, Kerala 673601, India.

${ }^{2}$ Associate Professor, Department of Electronics and Communication Engineering, National Institute of Technology, Calicut, Kerala 673601, India.

Received: 9 July 2016 Accepted: 4 August 2016

Published online: 17 August 2016

\section{References}

1. H Cao, V Leung, C Chow, H Chan, Enabling technologies for wireless body area networks: a survey and outlook. Commun. Mag. IEEE. 47(12), 84-93 (2009)

2. A Boulis, D Smith, D Miniutti, L Libman, Y Tselishchev, Challenges in body area networks for healthcare: the MAC. Commun. Mag. IEEE. 50(5), 100-106 (2012)

3. B Zhen, M Patel, S Lee, E Won, A Astrin, IEEE 802.15 TG6 technical requirements document (TRD) (2008). https://mentor.ieee.org/802.15/ documents?isdcn $=644 \&$ isgroup $=0006$

4. D Miniutti, D Smith, L Hanlen, A Zhang, A Boulis, D Rodda, B Gilbert, Sleeping channel measurements for body area networks. IEEE. $\mathbf{8 0 2}$ 15-09-0778-01-0006 (2010)

5. A Astrin, et al, IEEE standard for local and metropolitan area networks. Part 15.6: wireless body area networks. The document is available at IEEE Xplore. (IEEE, 2012)

6. C BoKeun, K ByungSoo, L SangSeol, W KyuYeul, K YongJun, C DuckJin, in Proc. of Pervasive Computing Signal Processing and Applications (PCSPA), 
International Conference on. Narrow band physical layer design for WBAN system, (IEEE Harbin Institute of Technology, Harbin, China, 2010), pp. 154-157

7. SS Haykin, Digital communications. (Wiley, New York, 1988)

8. A Kailas, in Proc. of IEEE conference on Engineering in Medicine and Biology Society. Power allocation strategies to minimize energy consumption in wireless body area networks (IEEE, Boston, 2011), pp. 2204-2207

9. X Huang, H Shan, X Shen, in Proc. of IEEE conference on Wireless Communications and Networking Conference. On energy efficiency of cooperative communications in wireless body area networks (IEEE, Mexico, 2011), pp. 1097-1101

10. KS Deepak, AV Babu, in Proc. of IEEE India Conference (INDICON). Packet size optimization for energy efficient cooperative wireless body area networks (IEEE, Kochi, India, 2012), pp. 736-741

11. KS Deepak, AV Babu, in Proc. of Advances in Computing, Communications and Informatics (ICACCI). Energy efficiency of IEEE 802.15.6 based wireless body area networks in scheduled access mode (IEEE, Mysore, India, 2013), pp. 301-307

12. GE Arrobo, RD Gitlin, in Proc. of IEEE conference on Engineering in Medicine and Biology Society. Improving the reliability of wireless body area networks (IEEE, Boston, 2011), pp. 2192-2195

13. JY Oh, JH Kim, HS Lee, JY Kim, PSSK modulation scheme for high data rate implantable medical devices. IEEE Trans. Inf. Technol. Biomed. 14(3), 634-640 (2010)

14. J Elias, Optimal design of energy-efficient and cost-effective wireless body area networks. Ad Hoc Netw. Elsevier. 13, 560-574 (2014)

15. X Zhou, T Zhang, L Song, Q Zhang. Proc. of IEEE VTC, vol. 5 (Spring, Seoul, Korea, 2014), pp. 1-6

16. Y Sankarasubramaniam, IF Akyildiz, SW McLaughlin, in Proc. of IEEE conference on Sensor Network Protocols and Applications. Energy efficiency based packet size optimization in wireless sensor networks, (Anchorage, AK, USA, 2003), pp. 1-8

17. S Ho, J Yeh, J Alexander, N Evgenios, K Sanghoek, T Yuji, PB Beygui, RE Poon, SY Ada, Wireless power transfer to deep-tissue microimplants. Proc. Natl. Acad. Sci. 111(22), 7974-7979 (2014)

18. K Sayrafian-Pour, WB Yang, J Hagedorn, J Terrill, KY Yazdandoost, in Proc. of IEEE Personal, Indoor and Mobile Radio Communications. A statistical path loss model for medical implant communication (IEEE, Tokyo, 2009), pp. 2995-2999

19. K Sayrafian-Pour, WB Yang, JG Hagedorn, JE Terrill, KY Yazdandoost, $K$ Hamaguchi, Channel models for medical implant communication. IJWIN. 17(3-4), 105-112 (2010)

20. J Hagedorn, J Terrill, W Yang, K Sayrafian, K Yazdandoost, R Kohno, A statistical path loss model for MICS. Citeseer, 802.15-08-0519-01-0006 (2008)

21. KY Yazdandoost, K Sayrafian-Pour, Channel model for body area network (BAN). (IEEE, 2009), pp. P802.15-08-0780-09-0006

22. T Aoyagi, J Takada, K Takizawa, N Katayama, T Kobayashi, KY Yazdandoost, $\mathrm{H}$ Li, R Kohno, Channel model or wearable and implantable WBANs. IEEE 802.15 WPAN Document IEEE P802. 15-08-0416-04-0006, 1-57 (2008)

23. E Reusens, W Joseph, B Latré, B Braem, G Vermeeren, E Tanghe, L Martens, I Moerman, C Blondia, Characterization of on-body communication channel and energy efficient topology design for wireless body area networks. IEEE Trans. Inf. Technol. Biomed. 13(6), 933-945 (2009)

24. A Fort, J Ryckaert, C Desset, P De Doncker, P Wambacq, L Van Biesen, Ultra-wideband channel model for communication around the human body. IEEE J. Selected Areas Commun. 24(4), 927-933 (2006)

25. N Wu, X Wang, H Dai, H Zhao, in Proc. of International Conference on Communications in China (ICCC). On the energy consumption of M-PSK modulation schemes under fading channels (IEEE, 2013), pp. 380-384

26. Zarlink, ZL70101. http://www.zarlink.com/zarlink/

27. Nordic, nRF24L01+. http://www.nordicsemi.com/

28. S Lin, DJ Costello, Error control coding, 2nd ed. (Prentice-hall, Englewood Cliffs, 2004)

\section{Submit your manuscript to a SpringerOpen ${ }^{\circ}$ journal and benefit from:}

- Convenient online submission

- Rigorous peer review

- Immediate publication on acceptance

- Open access: articles freely available online

- High visibility within the field

- Retaining the copyright to your article

Submit your next manuscript at $\gg$ springeropen.com 